\title{
Method of the Variations for the Solution of Optimal Control Problems
}

\author{
Mustafina Svetlana, Igor Grigoryev \\ Dr., Professor of Bashkir State University, Sterlitamak, Physics and Mathematics Department. Work address: Lenin \\ ave., 47-a, Sterlitamak, Russia, 453103 \\ Candidate for a Master's Degree, Bashkir State University, Sterlitamak, Physics and Mathematics Department \\ Work address: Lenin ave., 47-a, Sterlitamak, Russia
}

\begin{abstract}
This article deals with the main points of numerical solution of optimal control problems basing on the method of variations. We give a description of an algorithm of the method of variations a numerical solution of problems of optimal control. Finally, numerical examples are included to demonstrate efficiency, simplicity and high accuracy of the proposed method.
\end{abstract}

Keywords: Method Of Variations, Optimal Control, Phase Variables, Numerical Solution.

\section{INTRODUCTION}

The theory of optimal control has been well developed for over forty years. Control theory is application-oriented mathematics that deals with the basic principles underlying the analysis and design of (control) systems. Systems can be engineering systems (air conditioner, aircraft, and CD player etcetera), economic systems, biological systems and so on. To control means that one has to influence the behavior of the system in a desirable way: for example, in the case of an air conditioner, the aim is to control the temperature of a room and maintain it at a desired level, while in the case of an aircraft, we wish to control its altitude at each point of time so that it follows a desired trajectory.

As a result, more and more people will benefit greatly by learning to solve the optimal control problems numerically. Realizing such growing needs, books on optimal control put more weight on numerical methods. In retrospect, [1] was the first and the "classic" book for studying the theory as well as many interesting cases (timeoptimal, fuel-optimal and linear quadratic regulator problems). Necessary conditions for various systems were derived and explicit solutions were given when possible. Later, [2] proved to be a concise yet excellent book with more engineering examples.

\section{Problem Statement}

The formulation of an optimal control problem requires several steps: the class of admissible controls is discussed in 2.1; the mathematical description (or model) of the system to be controlled is considered in 2.2; then, the specification of a performance criterion is addressed in 2.3; Finally, we close the section with the statement of physical constraints that should be satisfied is described in 2.4 .

\subsection{Admissible Controls}

We shall consider the behaviour of a system whose state at any instant of time is characterized by $n \geq 1$ real numbers $x=\left(x_{1}, \ldots, x_{n}\right)^{T} \in R^{n}$ (for example, these may be coordinates and velocities). The vector space of the system under consideration is called the phase space. It is assumed that the system can be controlled, i.e., the system is equipped with controllers whose position dictates its future evolution. These controllers are characterized by points $u=\left(u_{1}, \ldots, u_{q}\right)^{T} \in U \subseteq R^{q}, q \geq 1$, namely the control variables.

In the vast majority of optimal control problems, the values that can be assumed by the control variables are restricted to a certain control region $\mathrm{U}$, which may be any set in $R^{q}$. In applications, the case where $\mathrm{U}$ is a closed region in $R^{q}$ is important. For example, the control region $\mathrm{U}$ may be a hypercube:

$\left|u_{j}\right| \leq 1, j=1, \ldots, q$. 


\section{American Research Journal of Mathematics, Volume 1, Issue 4, 2015 \\ ISSN 2378-704X}

The physical meaning of choosing a closed and bounded control region is clear. The quantity of fuel being supplied to a motor, temperature, current, voltage, etc., which cannot take on arbitrarily large values, may serve as control variables. More general relations, such as:

$\phi(u)=0$,

may also exist among the control variables.

We shall call every function $u(\cdot)$, defined on some time interval $t \in T=\left[t_{0}, t_{1}\right]$, a control. A control is an element of a (normed) linear space of real-vector-valued functions.

\subsection{Dynamical System}

A nontrivial part of any control problem is modelling the system. The objective is to obtain the simplest mathematical description that adequately predicts the response of the physical system to all admissible controls. We shall restrict our discussion herein to systems described by ordinary differential equations in state-space form:

$$
\frac{d x_{i}}{d t}=f_{i}\left(x_{1}, \ldots x_{n}, u_{1}, \ldots, u_{r}, t\right), x_{i}(0)=x_{i 0}, i=1, \ldots, n \text {. }
$$

Here, $t \in R$ stands for the independent variable, usually called time; in the case where $f$ does not depend explicitely on $t$, the system is said to be autonomous. The vector $u \in U \subseteq R^{q}$ represents the control (or input or manipulated) variables at time instant t. The vector $x(t) \in R^{n}, n \geq 1$, represents the state (or phase) variables, which characterize the behavior of the system at any time instant $\mathrm{t}$. A solution $\mathrm{x}(\mathrm{t}, \mathrm{x} 0, \mathrm{u}()$.$) of (3) is called a response$ of the system, corresponding to the control $u(\cdot)$, for the initial condition $x_{i}(0)=x_{i 0}, i=1, \ldots, n$.

So one can think of a control system as a box, which given the input $\mathrm{u}$ and intial state $x_{i}(0)=x_{i 0}, i=1, \ldots, n$, manufactures the state according to the law (3); see Figure 1.

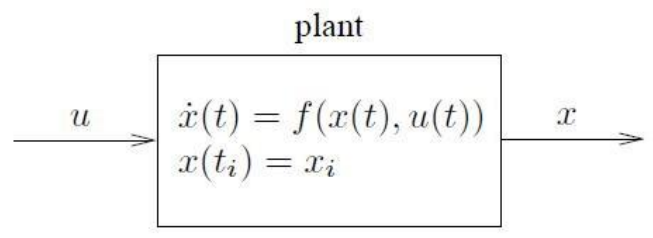

Fig1. A control system

\subsection{Performance Criterion}

A performance criterion (also called cost functional or simply cost) must be specified for evaluating the performance of a system quantitatively. By analogy to the problems of the calculus of variations, the cost functional $I: U\left[t_{0}, t_{1}\right] \rightarrow R$ may be defined in the so-called Lagrange form:

$I(u)=\int_{t_{0}}^{t_{1}} f^{0}(t, x(t), u(t)) d t$

\subsection{Physical Constraints}

A great variety of constraints may be imposed in an optimal control problem. These constraints restrict the range of values that can be assumed by both the control and the state variables. One usually distinguishes between point constraints and path constraints; optimal control problems may also contain isoperimetric constraints. All these constraints can be of equality or inequality type.

\subsubsection{Point Constraints.}

These constraints are used routinely in optimal control problems, especially terminal constraints (i.e., point constraints defined at terminal time). As just an example, an inequality terminal constraint of the form: 


\section{American Research Journal of Mathematics, Volume 1, Issue 4, 2015}

ISSN 2378-704X

$\psi\left(t_{1}, x\left(t_{1}\right)\right) \leq 0$,

May arise in stabilization problems, e.g., for forcing the system's response to belong to a given target set at terminal time; another typical example is that of a process changeover where the objective is to bring the system from its actual steady state to a new steady state:

$\psi^{\prime}\left(t_{1}, x\left(t_{1}\right)\right) \leq 0$.

\subsubsection{Isoperimetric Constraints.}

Like problems of the calculus of variations, optimal control problems may have constraints involving the integral of a given functional over the time interval $\left[t_{0}, t_{1}\right]$ (or some subinterval of it):

$\int_{t_{0}}^{t_{1}} h(t, x(t), u(t)) d t \leq C$

\subsubsection{Path Constraints.}

This last type of constraints is encountered in many optimal control problems. Path constraints may be defined for restricting the range of values taken by mixed functions of both the control and the state variables. Moreover, such restrictions can be imposed over the entire time interval $\left[t_{0}, t_{1}\right]$ or any (nonempty) time subinterval, e.g., for safety reasons. For example, a path constraint could be defined as:

$$
\phi(t, x(t), u(t)) \leq 0, \forall t \in\left[t_{0}, t_{1}\right]
$$

Hence restricting the points in phase space to a certain region $x(t) \in R^{n}$ at all times. In general, a distinction is made between those path constraints depending explicitly on the control variables, and those depending only on the state variables ("pure" state constraints) such as:

$x_{k}(t) \leq x^{U}, \forall t \in\left[t_{0}, t_{1}\right]$,

for some $k=\{1, \ldots, n\}$. This latter type of constraints being much more problematic to handle.

\section{THE ALGORITHM OF THE METHOD OF VARIATIONS}

The algorithm consists of 9 steps:

1. Guess an initial approximation of control $U_{0}$.

2. Break interval $\left[t_{0}, t_{k}\right]$ to $n$ parts, constituting a uniform system of units.

3. Select starting node $t_{0}$, which will be a variation of controls.

4. Compute $U\left(t_{0}\right) \pm \delta U$.

5. Compute $x(t), u(t)$ by solving (3).

6. Calculate $I(u)$ according to (4).

7. Go to $t_{1}$ and go to step 4 for all remaining points $t_{i}$.

8. Determine the minimum value of the criterion calculated for all points $t_{i}$ and define a new control $U_{1}$ corresponds to the lowest value criterion.

9. Set $\delta U=\frac{\delta U}{2}$. Then, with the $\operatorname{control} U_{1}$, go to step 3 until will not find variation in which the performance criterion will not be improved. 


\section{DISCUSSION}

The software for the numerical calculations presented below in this article was developed in Borland Delphi environment. For each of the following cases, we will compute the Euclidean norm of the solution error:

$$
\begin{aligned}
& \varepsilon_{x_{1}}=\sqrt{\sum_{i}\left(x_{1 i}-x_{1}^{*}\left(t_{i}\right)\right)^{2}}, \\
& \varepsilon_{x_{2}}=\sqrt{\sum_{i}\left(x_{2 i}-x_{2}^{*}\left(t_{i}\right)\right)^{2}} \\
& \varepsilon_{u}=\sqrt{\sum_{i}\left(u_{i}-u^{*}\left(t_{i}\right)\right)^{2}} .
\end{aligned}
$$

Example 1. Consider the following optimal control problem:

$$
\begin{aligned}
& \left\{\begin{array}{l}
\dot{x}_{1}(t)=x_{2}(t), \\
\dot{x}_{2}(t)=-x_{1}(t)+u(t) ;
\end{array}\right. \\
& x_{1}(0)=0, x_{2}(0)=0, \\
& 0 \leq t \leq 2 \pi, \\
& |u| \leq 1 .
\end{aligned}
$$

The performance measure is:

$$
I\left(x_{1}, x_{2}\right)=x_{2}(2 \pi) \rightarrow \min .
$$

The optimal control problem is to find a control law $u^{*}(\cdot)$ which minimizes cost functional (14)

The necessary conditions, derived from calculus of variations, can be solved analytically; and we obtain for the state and control variables of the optimal trajectory in $0 \leq t<2 \pi$ :

$$
u^{*}(t)=\left\{\begin{array}{l}
-1,0 \leq t<\frac{\pi}{2} \\
1, \frac{\pi}{2} \leq t<\frac{3 \pi}{2} \\
-1, \frac{3 \pi}{2} \leq t<2 \pi
\end{array}\right.
$$$$
x_{1}^{*}(t)=\left\{\begin{array}{l}
\cos t-1,0 \leq t<\frac{\pi}{2}, \\
\cos t-2 \sin t+1, \frac{\pi}{2} \leq t<\frac{3 \pi}{2}, \\
\cos t-4 \sin t-1, \frac{3 \pi}{2} \leq t<2 \pi .
\end{array}\right.
$$

$$
x_{2}{ }^{*}(t)=\left\{\begin{array}{l}
-\sin t, 0 \leq t<\frac{\pi}{2}, \\
-\sin t-2 \cos t, \frac{\pi}{2} \leq t<\frac{3 \pi}{2}, \\
-\sin t-4 \cos t, \frac{3 \pi}{2} \leq t<2 \pi .
\end{array}\right.
$$

The minimum functional value of is $x_{2}{ }^{*}(2 \pi)=-4$. 


\section{American Research Journal of Mathematics, Volume 1, Issue 4, 2015}

ISSN 2378-704X

Fig. 2 - Fig. 3 shows the comparison between numerical solution and approximate solution for $u_{0}=0.3$. It can be noticed that curves are in good agreement. Next, we found simulation results for different initial guess and accuracy of this problem; see Table 1.

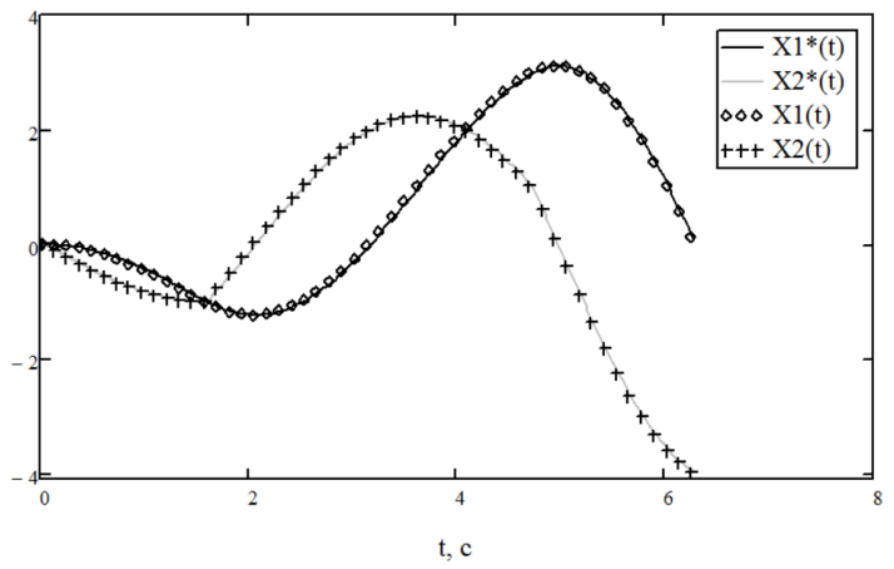

Fig2. The suboptimal states, Example 1

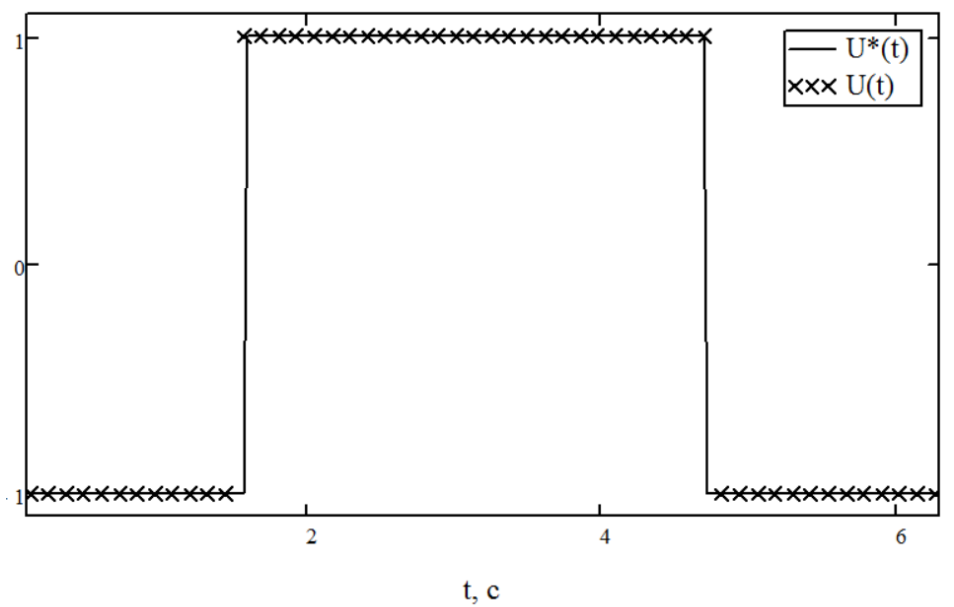

Fig3. The suboptimal control, Example 1

Table1. Simulation results for different initial guess and accuracy, Example 1

\begin{tabular}{|l|l|l|l|l|l|l|l|}
\hline № & $u_{0}$ & Accuracy & Elapsed time, s. & $\varepsilon_{u}$ & $\varepsilon_{x_{1}}$ & $\varepsilon_{x_{2}}$ & $I_{\min }$ \\
\hline 1 & 0 & 0,1 & 2,06 & 3,06 & 1,11 & 1,21 & $-3,74$ \\
\hline 2 & 0 & 0,01 & 2,85 & 2,99 & 0,14 & 0,15 & $-3,97$ \\
\hline 3 & 0 & 0,001 & 4,12 & 2,987 & 0,018 & 0,019 & $-3,9949$ \\
\hline 4 & $-0,6$ & 0,001 & 3,94 & 2,854 & 0,019 & 0,016 & $-3,9958$ \\
\hline 5 & $-0,9$ & 0,0001 & 12,06 & 2,0024 & 0,1086 & 0,1089 & $-3,9994$ \\
\hline 6 & 0,1 & 0,00001 & 23,35 & 2,0023 & 0,1093 & 0,1088 & $-3,9997$ \\
\hline
\end{tabular}

Example 2. Consider the following optimal control system:

$$
\begin{aligned}
& \left\{\begin{array}{l}
\dot{x}_{1}(t)=x_{2}(t), \\
\dot{x}_{2}(t)=u(t)
\end{array}\right. \\
& x_{1}(0)=-1, x_{2}(0)=0, \\
& 0 \leq t \leq 2.5,
\end{aligned}
$$


American Research Journal of Mathematics, Volume 1, Issue 4, 2015

ISSN 2378-704X

$|u| \leq 1, x_{2} \leq 0.5$.

The performance measure is:

$I\left(x_{1}, x_{2}\right)=x_{1}^{2}(2.5)+x_{2}^{2}(2.5) \rightarrow \min$.

The optimal control problem is to find a control law $u^{*}(\cdot)$ which minimizes cost functional (19)

The exact solution is:

$u^{*}(t)=\left\{\begin{array}{l}1, t \leq 0.5, \\ 0,0.5<t<2, \\ -1,2 \leq t \leq 2.5 .\end{array}\right.$

Fig. 4 - Fig. 5 shows the comparison between numerical solution and approximate solution for $u_{0}=0.1$. Table 2 presents simulation results for different initial guess and accuracy of this problem.

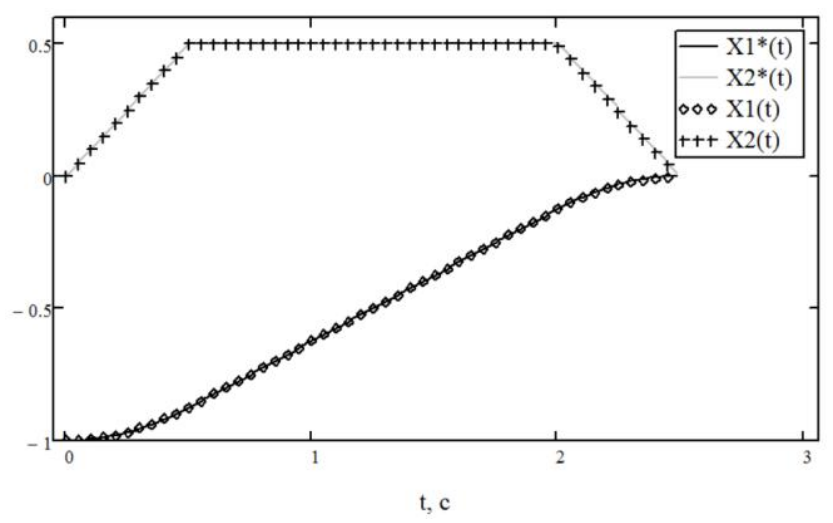

Fig4. The suboptimal states, Example 2

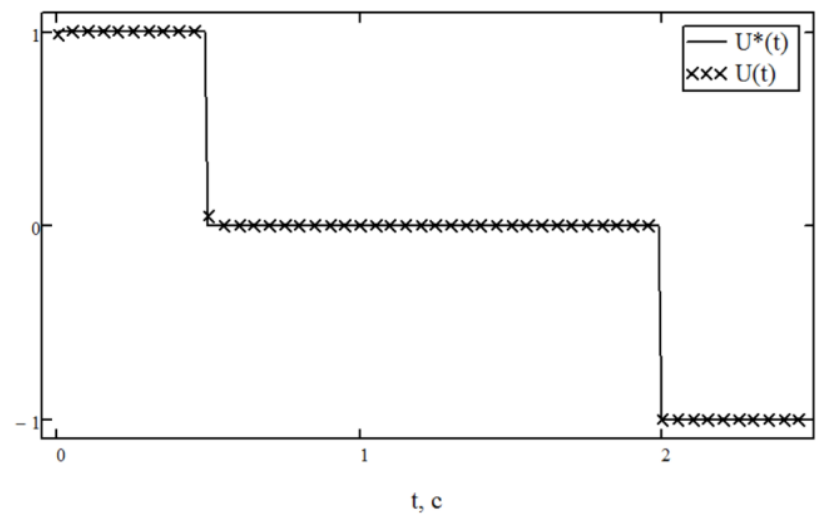

Fig5. The suboptimal control, Example 2

Table2. Simulation results for different initial guess and accuracy, Example 2

\begin{tabular}{|l|l|l|l|l|l|l|l|}
\hline № & $u_{0}$ & Accuracy & Elapsed time, s. & $\varepsilon_{u}$ & $\varepsilon_{x_{1}}$ & $\varepsilon_{x_{2}}$ & $I_{\min }$ \\
\hline 1 & 0 & 0,1 & 1,12 & 5,46 & 2,97 & 2,58 & 0,15 \\
\hline 2 & 0 & 0,01 & 3,41 & 1,79 & 0,09 & 0,102 & 0,0001 \\
\hline 3 & 0 & 0,001 & 3,95 & 1,807 & 0,088 & 0,102 & 0,0001 \\
\hline 4 & $-0,6$ & 0,001 & 4,34 & 1,695 & 0,096 & 0,102 & 0,0001 \\
\hline 5 & $-0,9$ & 0,0001 & 7,98 & 1,5092 & 0,0885 & 0,1026 & 0,0001 \\
\hline 6 & 0,1 & 0,00001 & 13,48 & 1,50543 & 0,08875 & 0,10224 & 0,00014 \\
\hline
\end{tabular}




\section{American Research Journal of Mathematics, Volume 1, Issue 4, 2015}

ISSN 2378-704X

\section{CONCLUSION}

For many optimal control problems, the method of variations is the best option we have. The advantage of this algorithm is that it does not have requirements with initial guess. The algorithm has good convergence and can be used to solve a large class of applications in various fields of national economy.

\section{REFERENCES}

[1] Athans M. and Falb P. Optimal control: an introduction to the theory and its applications. Dover Publications, Inc. 2007.

[2] Kirk D. E. Optimal control theory: an introduction. Dover Publications,

[3] Inc. 2004.

[4] Wang X. Solving Optimal Control Problems with MATLAB-Indirect Methods. 2009.

[5] Fedorenko R. P. Approximate solution of optimal control problems. Moscow, Nauka, 1978, 488 p.

[6] Grigoryev I., Mikhaylova T., Mustafina S. About numerical algorithm method of variation in the control area // Fundamental research. 2015. № 5-2. pp. 279-283.

[7] Grigoryev I., Mustafina S. Implementation of numerical algorithm for solving optimal control problems with phase constraints // Postgraduate Student. 2015. № 5-1 (10). pp. 49-51.

[8] Grigoryev I., Mustafina S. Finding an optimal programmed control by iteration method // The Way of Science. 2015. № 5 (15). pp. 10-13.

[9] Mustafina S.A., Balaev A.V., Smirnov D.Y., Spivak S.I. Modeling of the Catalytic Process of Dehydrogenation of Methyl Butenes. // Control Systems and Information Technology, 2006, vol. 1(23), pp. $10-14$.

[10] Mustafina S.A., Valieva Yu.A., Davletshin R.S., Balaev A.V., Spivak S.I. Optimum Technological Decisions for Catalytic Processes and Reactors. // Kinetika i Kataliz, 2005, vol. 46, no. 5, pp. 749-756.

[11] Ostrovsky G., Volin Yu.M. Methods for optimization of complex chemical-engineering schemes. Moscow, Chemistry, 1970. 328 p.

[12] Panteleev A.V. Bortakovskii A.S., Letova T.A. Optimal control in the examples and problems. / M .: MAI, 1996. $-212 \mathrm{p}$.

[13] Pontryagin L.S. The mathematical theory of optimal processes. -M.:Nauka, 1976. - 392 p. 